\begin{tabular}{|c|l|}
\hline Title & Harmonic-oscillator excitations of precise few-body wave functions \\
\hline Author(s) & Horiuchi, W.; Suzuki, Y. \\
\hline Citation & $\begin{array}{l}\text { Physical Review C, 90(3), 34001 } \\
\text { https://doi.org/_0.1103/PhysRevC.90.034001 }\end{array}$ \\
\hline Issue Date & 2014_09-02 \\
\hline Doc URL & http://hdl.handle.net/2115/57075 \\
\hline Rights & O2014 A merican Physical Society \\
\hline Type & article \\
\hline File Information & PhysRevC_90_034001-.pdf \\
\hline
\end{tabular}

Instructions for use 


\title{
Harmonic-oscillator excitations of precise few-body wave functions
}

\author{
W. Horiuchi ${ }^{1}$ and Y. Suzuki ${ }^{2,3}$ \\ ${ }^{1}$ Department of Physics, Hokkaido University, Sapporo 060-0810, Japan \\ ${ }^{2}$ Department of Physics, Niigata University, Niigata 950-2181, Japan \\ ${ }^{3}$ RIKEN Nishina Center, Wako 351-0198, Japan \\ (Received 5 June 2014; published 2 September 2014)
}

\begin{abstract}
A method for calculating the occupation probability of the number of harmonic-oscillator (HO) quanta is developed for a precise few-body wave function obtained in a correlated Gaussian basis. The probability distributions of two- to four-nucleon wave functions obtained by using different nucleon-nucleon $(N N)$ interactions are analyzed to gain insight into the characteristic behavior of the various interactions. Tensor correlations as well as short-range correlations play a crucial role in enhancing the probability of high $\mathrm{HO}$ excitations. For the excited states of ${ }^{4} \mathrm{He}$, the interaction dependence is much less because high $\mathrm{HO}$ quanta are mainly responsible for describing the relative-motion function between the $3 N+N\left({ }^{3} \mathrm{H}+p\right.$ and $\left.{ }^{3} \mathrm{He}+n\right)$ clusters.
\end{abstract}

DOI: 10.1103/PhysRevC.90.034001

PACS number(s): 21.60.De, 21.30.-x, 27.10.+h

\section{INTRODUCTION}

The nuclear shell model is a standard microscopic theory for describing a many-nucleon system. For doubly closed nuclei, first we consider the lowest harmonic-oscillator (HO) state expressed with a single Slater determinant (SD), an antisymmetrized product of single-particle $\mathrm{HO}$ orbits. To take many-body correlations into account, multiparticle-multihole $(m \mathrm{p}-m \mathrm{~h})$ configuration-mixing calculations are performed by superposing many $\mathrm{SD}$ states that include higher $\mathrm{HO}$ excitations.

Thus far, the ab initio no-core shell model (NCSM) with modern nuclear forces has been developed extensively [1]. In the NCSM, all nucleons are active, but a crucial approximation is the truncation of maximum HO quanta, which determines the NCSM space. Compared to ordinary shell-model effective interactions, the use of realistic nuclear forces requires many SD states in higher major shells to reach convergence because of strong couplings between low- and high-momentum components arising from the tensor component and short-range repulsion of the nuclear force.

The HO expansion provides us with systematic and sizeextensive calculations, but it is not advantageous to describe the spatial extent of the wave function because of a rapid falloff of the $\mathrm{HO}$ wave functions. If a system exhibits a cluster structure, the subsystems are localized and their relativemotion wave function can have a long tail. For example, the first-excited state of ${ }^{16} \mathrm{O}$ is still difficult to reproduce by present large-scale shell-model calculations [2,3]. As shown in Refs. [4-7], the cluster structure always induces extremely high $\mathrm{HO}$ quanta beyond the limitation of present computational resources. To make an efficient description of nuclear manybody systems using the HO expansion, it is useful to know how many $\mathrm{HO}$ excitations are required to account for those important correlations which are induced by the tensor force, the short-range repulsion, and the cluster structure.

Nowadays, precise wave functions of very light nuclei can be obtained by using ab initio few-body methods. Although the few-body method can only be applied to very light nuclei, long-ranged asymptotics as well as short-range correlations are accurately described. Such few-body wave functions can provide important hints on how to tackle complicated manybody problems. Since the shell model and the few-body model are formulated in different frameworks, it is convenient to relate the few-body wave function to the $\mathrm{HO}$ wave function. For this purpose, by extending the formulation of Ref. [5], we calculate the occupation probability of the number of total $\mathrm{HO}$ quanta in the wave function obtained with an $a b$ initio few-body method; in particular, by using a correlated Gaussian (CG) basis with global vectors [8-11]. We calculate the HO occupation probability of the wave functions of two- to fournucleon systems and discuss its properties, especially focusing on the ${ }^{4} \mathrm{He}$ system.

The nucleus ${ }^{4} \mathrm{He}$ is an interesting example because important ingredients of many-nucleon dynamics show up in its spectrum. The ground state is strongly correlated due to the tensor component and short-range repulsion of the nuclear force. The tensor force induces a $D$-state mixing of approximately 14\% [12]. Since the two nucleons cannot overlap with each other due to the short-range repulsion, the universal short-range behavior is found in the pair correlation functions $[13,14]$. In the excited states, the structure changes drastically. The first excited $0_{2}^{+}$state of ${ }^{4} \mathrm{He}$ is recognized to have a ${ }^{3} \mathrm{H}+p$ and ${ }^{3} \mathrm{He}+n(3 N+N)$ cluster structure with $S$-wave relative motion [15]. The negative-parity states are located at just a few $\mathrm{MeV}$ above the $0_{2}^{+}$state and are considered to be parity-inverted partners of the $0_{2}^{+}$state. They have the intrinsic structure of $3 N+N$ with $P$-wave relative motion [16]. Thus, ${ }^{4} \mathrm{He}$ has a similarity to ${ }^{16} \mathrm{O}$ [4] that exhibits a ${ }^{12} \mathrm{C}+\alpha$ cluster structure in its spectrum.

The analysis of the oscillator excitations will be useful for developing and improving a truncation scheme for the model space in, e.g., ab initio NCSM [1], importance-truncated NCSM [17,18], the symmetry-adapted no-core shell model [19], the Monte Carlo shell model [20], as well as a model approach such as the tensor-optimized shell model [21].

The paper is organized as follows: Section II A gives a basic formula to calculate the occupation probability of the $\mathrm{HO}$ 
quanta in the CG basis. Section II B defines the Hamiltonian and the CG basis and briefly explains how we obtain the precise few-body wave functions. Section III demonstrates the HO occupation probability distributions for two- to four-nucleon systems. Four different potentials are employed in order to examine how the $\mathrm{HO}$ distribution reflects the characteristics of the nuclear force. In Sec. III A, we discuss the role of the HO quanta higher than the lowest $(0 s)^{N}$ configuration, focusing on the relationship with the short-range repulsion and tensor correlations in the ground state of ${ }^{4} \mathrm{He}$. Long-range cluster correlations in the excited states of ${ }^{4} \mathrm{He}$ are discussed in Sec. III B. Section III C discusses the parity-inverted partners of the first-excited state of ${ }^{4} \mathrm{He}$. The summary is given in Sec. IV. An Appendix details a method for calculating the probability of the HO quanta.

\section{FORMULATION}

\section{A. Harmonic-oscillator occupation probability}

Let $\Psi$ denote an $N$-nucleon wave function that is free of spurious center-of-mass (c.m.) motion. The occupation probability $P_{Q}$ of the $Q \hbar \omega$ components in $\Psi$ is calculated using an integral of the projection-operator type

$$
P_{Q}=\frac{1}{2 \pi} \int_{0}^{2 \pi} d \theta e^{-i Q \theta}\left\langle\Psi\left|e^{i \theta \mathcal{O}}\right| \Psi\right\rangle,
$$

where $\mathcal{O}$ is an operator that counts the number of $\mathrm{HO}$ quanta:

$$
\mathcal{O}=\sum_{i=1}^{N-1}\left(\frac{1}{\hbar \omega} H_{\mathrm{HO}}(i)-\frac{3}{2}\right) \text {. }
$$

Here $H_{\mathrm{HO}}(i)$ is the $\mathrm{HO}$ Hamiltonian for the $i$ th relative coordinate. See the Appendix for details. The method for calculating $P_{Q}$ was developed for microscopic multicluster wave functions in Ref. [5]. We extend it to the case where $\Psi$ is given in terms of a combination of $\mathrm{CG}$ basis functions.

The CG is constructed from the generating function $[8,9]$

$$
g(\boldsymbol{s} ; A, \boldsymbol{x})=\exp \left(-\frac{1}{2} \tilde{\boldsymbol{x}} A \boldsymbol{x}+\tilde{\boldsymbol{s}} \boldsymbol{x}\right),
$$

where the $(N-1) \times(N-1)$ matrix $A$ is positive definite and symmetric, $s$ is a column vector of dimension $(N-1)$ to describe the angular motion of the system, and $\boldsymbol{x}$ is a column vector of dimension $(N-1)$ whose element is the three-dimensional relative coordinate $\boldsymbol{x}_{i}$. The transpose of a matrix is indicated by a tilde symbol. Both $A$ and $s$ are variational parameters, which makes the CG flexible and easily adapted to few-body problems. To calculate the quantity (1) with the $\mathrm{CG}$, we start from the matrix element of $e^{i \theta \mathcal{O}}$ between the generating functions (3). As detailed in the Appendix, the required matrix element reads

$$
\begin{aligned}
& \left\langle g\left(\boldsymbol{s}^{\prime} ; A^{\prime}, \boldsymbol{x}\right)\left|e^{i \theta \mathcal{O}}\right| g(\boldsymbol{s} ; A, \boldsymbol{x})\right\rangle \\
& \quad=\left(\frac{(2 \pi)^{N-1} \operatorname{det} \Gamma}{\operatorname{det} B \operatorname{det} C}\right)^{\frac{3}{2}} \exp \left(-\frac{1}{2} \tilde{\boldsymbol{s}} G \boldsymbol{s}+\frac{1}{2} \tilde{\boldsymbol{v}} B^{-1} \boldsymbol{v}\right),
\end{aligned}
$$

where $B=D+A^{\prime}, \boldsymbol{v}=z \Gamma C^{-1} \boldsymbol{s}+\boldsymbol{s}^{\prime}$, and $z=e^{i \theta}$. The matrices $\Gamma, C, D$, and $G$ are defined in the Appendix. Once the matrix element between the $\mathrm{CG}$ bases is obtained as a function of $\theta$, the integration in Eq. (1) is performed numerically.

\section{B. Few-body wave functions}

\section{Hamiltonian}

The Hamiltonian of the $N$-nucleon system is composed of the kinetic energy, two-body $N N$ interaction, and three-body interaction $(3 \mathrm{NF})$ terms

$$
H=\sum_{i=1}^{N} T_{i}-T_{\text {c.m. }}+\sum_{i<j} v_{i j}+\sum_{i<j<k} v_{i j k} .
$$

The c.m. kinetic energy is subtracted and no spurious c.m. motion is involved in the calculation. The inputs used in this paper are $\hbar^{2} / m=41.47106 \mathrm{MeV} \mathrm{fm}^{2}$ and $e^{2}=1.440 \mathrm{MeV} \mathrm{fm}$. The proton mass and neutron mass are taken to be equal.

We adopt (i) Minnesota (MN) [22], (ii) Afnan-Tang S3 (ATS3) [23], (iii) G3RS [24], and (iv) AV8' [25] potential models as the $N N$ interaction. A central 3NF [15] is added together with the realistic G3RS and $\mathrm{AV}^{\prime}$ potentials in order to reproduce the binding energies of ${ }^{3} \mathrm{H}$ and ${ }^{4} \mathrm{He}$. The MN potential is often used in microscopic cluster-model calculations. Although it has only a central term, the potential reproduces the binding energies of $N=2$ to 6 systems fairly well [8]. The ATS3 potential also has only the central term but contains a strong short-range repulsive core. The AV8' potential consists of central, spin-orbit, and tensor components, and has a strong short-range repulsion. The G3RS potential is somewhat softer than $\mathrm{AV}^{\prime}$ and gives a smaller $D$-state probability. The $\boldsymbol{L}^{2}$ and quadratic $\boldsymbol{L} \cdot \boldsymbol{S}$ terms in the G3RS potential are ignored.

\section{Correlated Gaussians and global vectors}

The wave function $\Psi$ is given as a combination of the basis functions expressed in the $L S$ coupling scheme

$$
\Phi_{(L S) J M_{J} T M_{T}}=\mathcal{A}\left[F_{L}(\boldsymbol{x}) \chi_{S}^{(\text {spin })}\right]_{J M_{J}} \eta_{T M_{T}}^{\text {(isospin) }},
$$

where $\mathcal{A}$ is the antisymmetrizer, and the square brackets [...] denote the angular-momentum coupling. The spin function is given in a successive coupling scheme

$$
\chi_{S M_{S}}^{(\text {spin) }}=\left[\cdots\left[\left[\chi_{1 / 2}(1) \chi_{1 / 2}(2)\right]_{S_{12}} \chi_{1 / 2}(3)\right]_{S_{123}} \cdots\right]_{S M_{S}} .
$$

The isospin wave function has exactly the same form as the spin part. All possible intermediate spins and isospins are included in the basis set. The orbital part is represented by the CG with two global vectors

$$
\begin{aligned}
& F_{\left(L_{1} L_{2}\right) L M_{L}}\left(u_{1}, u_{2}, A, \boldsymbol{x}\right) \\
& \quad=\exp \left(-\frac{1}{2} \tilde{\boldsymbol{x}} A \boldsymbol{x}\right)\left[\mathcal{Y}_{L_{1}}\left(\tilde{u}_{1} \boldsymbol{x}\right) \mathcal{Y}_{L_{2}}\left(\tilde{u}_{2} \boldsymbol{x}\right)\right]_{L M_{L}}
\end{aligned}
$$

with a solid harmonic

$$
\mathcal{Y}_{\ell m}(\boldsymbol{r})=r^{\ell} Y_{\ell m}(\hat{\boldsymbol{r}}),
$$

where $u_{i}$ is an $(N-1)$-dimensional column vector and $\tilde{u}_{i} \boldsymbol{x}$ is called a global vector that describes the rotational motion of the system. The off-diagonal matrix elements of $A$ explicitly describe correlations among the particles. The matrix element of the Hamiltonian between the CG of Eq. (8) can be obtained analytically from the one between the generating functions (3) in a systematic, algebraic procedure prescribed in Refs. [9-11]. The CG basis (8) has the great advantage that its functional form remains unchanged under a coordinate 
transformation, thereby easily facilitating operations such as those involved in channel rearrangements and permutations, etc. This flexibility enables us to apply the CG approach to many quantum-mechanical few-body problems. See Ref. [26] for various recent applications of the CG.

The ground states of ${ }^{2,3} \mathrm{H},{ }^{4} \mathrm{He}$ and the excited states of ${ }^{4} \mathrm{He}$ are obtained by using the stochastic variational method $[8,9]$. Although all the excited states of ${ }^{4} \mathrm{He}$ are above the ${ }^{3} \mathrm{H}+p$ threshold, we describe them in the square-integrable CG basis functions. Since they have relatively small decay widths ranging from 0.5 to $2 \mathrm{MeV}$ [27], the bound-state approximation works reasonably well as discussed in Ref. [28]. More details of calculations are given in Refs. [10,16,29].

\section{RESULTS AND DISCUSSIONS}

\section{A. Ground states: tensor and short-range correlations}

Figure 1 displays the probability of the lowest HO quantum $P_{0}$ for the ground state of ${ }^{4} \mathrm{He}$ as a function of the oscillator frequency $\hbar \omega$. We see moderate $\hbar \omega$ dependence of $P_{0}$ in all the potential models. Since the $P_{Q}$ distribution depends on $\hbar \omega$, we fix it by requiring that the lowest shell-model configuration, $(0 s)^{N}$, for the fixed $\hbar \omega$ reproduces the root-mean-square (rms) matter radius of the precise wave function. This is reasonable because the $(0 s)^{N}$ configuration is the dominant component of the wave function for $s$-shell nuclei. The $\hbar \omega$ values determined for ${ }^{4} \mathrm{He}$ are 23.2, 23.4, 22.2, and 21.6 MeV for MN, ATS3, $\mathrm{G} 3 \mathrm{RS}+3 \mathrm{NF}$, and AV8' $+3 \mathrm{NF}$ potentials, respectively. The $P_{0}$ values calculated with these $\hbar \omega$ values are close to the maximum values in Fig. 1.

Table I summarizes the calculated energy $E$, rms matter radius $r_{m}, D$-state probability $P(D)$, and $P_{0}$ of the ground state of ${ }^{2} \mathrm{H},{ }^{3} \mathrm{H}$, and ${ }^{4} \mathrm{He}$ for the different potential models. All the interactions give approximately the same $E$ and $r_{m}$ but quite different $P(D)$. The MN potential, which is the softest among the four potentials, gives the largest $P_{0}$ of approximately $95 \%$ for ${ }^{4} \mathrm{He}$. The $N=2$ to 4 wave functions with the MN potential are well described by the $(0 s)^{N}$ configurations. When the other

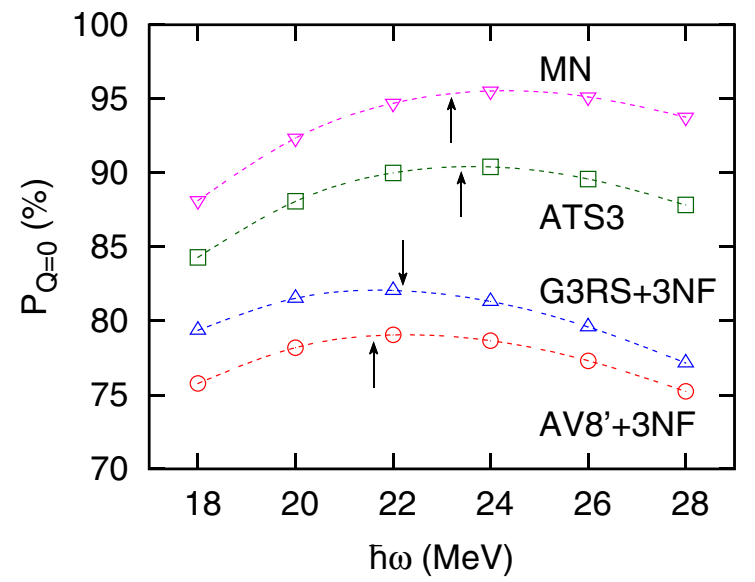

FIG. 1. (Color online) $\hbar \omega$ dependence of the occupation probability of the lowest $\mathrm{HO}$ quantum in the ground state of ${ }^{4} \mathrm{He}$. The arrow indicates the $\hbar \omega$ value that is employed to calculate $P_{Q}$ values of ${ }^{4} \mathrm{He}$. See text for detail.
TABLE I. Ground-state energies $(E)$ and rms matter radii $\left(r_{m}\right)$ of two- to four-nucleon systems calculated with different potential models: (i) $\mathrm{MN}$, (ii) $\mathrm{ATS} 3$, (iii) G3RS $+3 \mathrm{NF}$, and (iv) $\mathrm{AV} 8^{\prime}+$ $3 \mathrm{NF}$ potentials. The $D$-state probability $P(D)$ and the occupation probability $P_{0}$ of the $0 \hbar \omega$ component are given in percent. $M_{Q}$ and $\sigma_{Q}$ denote the average and standard deviation of the $P_{Q}$ distribution.

\begin{tabular}{lrrrcccc}
\hline \hline & & $E(\mathrm{MeV})$ & $r_{m}(\mathrm{fm})$ & $P(D)(\%)$ & $P_{0}(\%)$ & $M_{Q}$ & $\sigma_{Q}$ \\
\hline${ }^{2} \mathrm{H}$ & (i) & -2.20 & 1.95 & 0.00 & 89.6 & 0.534 & 1.95 \\
$\left(1^{+} 0\right)$ & (ii) & -2.22 & 1.94 & 0.00 & 89.4 & 0.692 & 3.77 \\
& (iii) & -2.28 & 1.98 & 4.78 & 86.9 & 1.27 & 5.80 \\
& (iv) & -2.24 & 1.96 & 5.77 & 85.5 & 1.57 & 6.84 \\
${ }^{3} \mathrm{H}$ & (i) & -8.38 & 1.71 & 0.00 & 90.8 & 0.409 & 1.70 \\
$\left(\frac{1}{2}{ }^{+} \frac{1}{2}\right)$ & (ii) & -8.76 & 1.67 & 0.00 & 89.7 & 0.787 & 3.99 \\
& (iii) & -8.35 & 1.74 & 7.10 & 84.9 & 1.52 & 5.96 \\
& (iv) & -8.41 & 1.70 & 8.69 & 83.1 & 1.92 & 7.08 \\
${ }^{4} \mathrm{He}$ & (i) & -29.94 & 1.41 & 0.00 & 95.4 & 0.263 & 1.48 \\
$\left(0_{1}^{+} 0\right)$ & (ii) & -30.83 & 1.42 & 0.00 & 90.4 & 0.934 & 4.00 \\
& (iii) & -28.56 & 1.47 & 11.42 & 82.1 & 1.96 & 5.98 \\
& (iv) & -28.43 & 1.45 & 14.07 & 79.1 & 2.59 & 7.31 \\
\hline \hline & & & & & & & \\
\hline
\end{tabular}

interactions are employed, the mixing of higher- $Q$ components becomes important. When a realistic potential is used, the deviation from the $(0 s)^{N}$ structure is the largest in ${ }^{4} \mathrm{He}$, which is the most tightly bound and has the largest $D$-state probability, as a result of the effects of short-range and tensor correlations. The ground state of ${ }^{4} \mathrm{He}$ obtained with the $\mathrm{AV} 8^{\prime}+3 \mathrm{NF}$ interaction predicts at most $80 \%$ of the $(0 s)^{4}$ configuration.

Figure 2 plots $P_{Q}(Q>0)$ of ${ }^{4} \mathrm{He}$. Consistently with the $M_{Q}$ and $\sigma_{Q}$ values in Table I, a harder interaction leads to $P_{Q \neq 0}$ that is more enhanced and extended to larger $Q$. In the case of the MN potential, $P_{Q}$ is found to be about $1 \%$ to $2 \%$ for $Q=2$ and 4, but it diminishes rapidly with increasing $Q$. Since no short-range repulsion is present in the MN potential, the configurations contributing to $P_{2}$ and $P_{4}$, e.g., $(0 s)^{3}(1 s)$ for $Q=2$ and $(0 s)^{2}(1 s)^{2},(0 s)^{3}(2 s)$ for $Q=4$, are expected to improve the tail of the wave function that cannot be described with $(0 s)^{4}$ alone. One may wonder why $P_{2}$ is smaller than

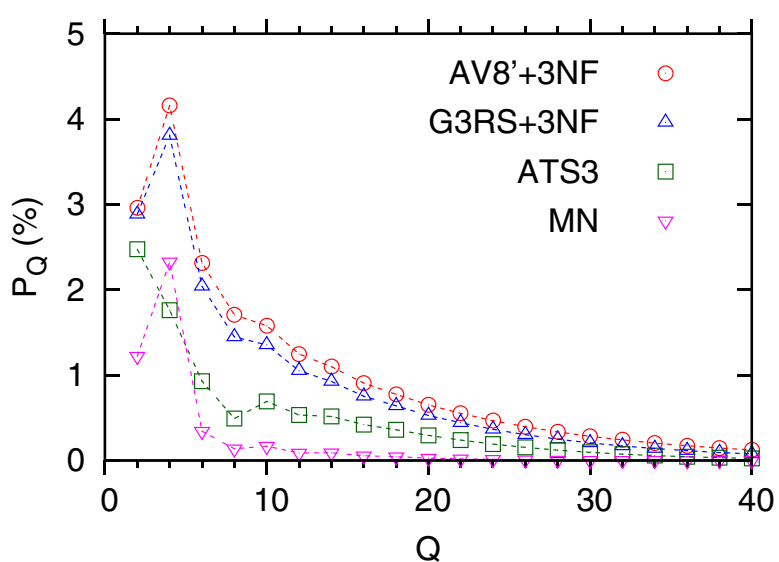

FIG. 2. (Color online) Occupation probability distributions of the total HO quanta for the ground state of ${ }^{4} \mathrm{He}$ calculated with different potential models. $P_{0}$ is not shown in the figure but given in Table I. $P_{Q}$ values are connected by dotted lines to guide the eye. 
$P_{4}$. We recalculate $P_{Q}$ by using smaller $\hbar \omega$ to describe the tail part more efficiently. For $\hbar \omega$ less than $20 \mathrm{MeV}$, the $P_{Q}$ distribution shows a monotonic decrease with increasing $Q$. The $P_{Q}$ values for small $Q$ depend on the choice of $\hbar \omega$. We will discuss this later in this section. With the ATS 3 potential, $P_{Q}$ decreases monotonically up to $Q=8$ and exhibits a bump at $Q=10$ with a long tail extending to more than $Q=30$, which is apparently due to the short-range repulsion. The G3RS + $3 \mathrm{NF}$ and $\mathrm{AV} 8^{\prime}+3 \mathrm{NF}$ potentials give a very similar pattern characterized by large and very extended $P_{Q}$ distributions. The probability is still $1.6 \%$ at $Q=10$ and $0.7 \%$ at $Q=20$ when the $\mathrm{AV} 8^{\prime}+3 \mathrm{NF}$ potential is used.

To discuss whether the short-range repulsion or the tensor component in the $N N$ interaction is important in determining the $P_{Q}$ distribution, we decompose $P_{Q}$ according to the total orbital angular momentum $L$. The ground-state wave function of ${ }^{4} \mathrm{He}$ is expressed in the notation of Eq. (6) as

$$
\Psi=\sum_{L=0,1,2} \sum_{i} C_{L}^{(i)} \Phi_{(L L) 0000}^{(i)}
$$

where the amplitude $C_{L}^{(i)}$ of the $i$ th basis state $\Phi_{(L L) 0000}^{(i)}$ satisfies $\sum_{L=0,1,2} \sum_{i}\left(C_{L}^{(i)}\right)^{2}=1$. The $P_{Q}$ is decomposed to a sum of $P_{Q}^{L}$ that is defined by

$$
\begin{aligned}
P_{Q}^{L}= & \frac{1}{2 \pi} \sum_{i}\left(C_{L}^{(i)}\right)^{2} \int_{0}^{2 \pi} d \theta e^{-i Q \theta} \\
& \times\left\langle\Phi_{(L L) 0000}^{(i)}\left|e^{i \theta \mathcal{O}}\right| \Phi_{(L L) 0000}^{(i)}\right\rangle
\end{aligned}
$$

Figure 3 displays $P_{Q}^{L}$ of the ground state of ${ }^{4} \mathrm{He}$ calculated with the $\mathrm{AV} 8^{\prime}+3 \mathrm{NF}$ potential. The $P_{Q}^{1}$ is negligibly small because the $L=1$ component occupies only $0.37 \%$ of the total wave function [29]. The $L=2$ component can couple with the $L=0$ configurations through the tensor force that induces a major shell mixing in the wave function. The $P_{Q}^{2}$ dominates up to $Q=18$, where the $P_{Q}^{0}$ gives an equal contribution. The $P_{Q}^{0}$ distribution shows a bump at $Q=10$ with a long tail, similarly to the ATS3-potential case. This suggests that the bump and

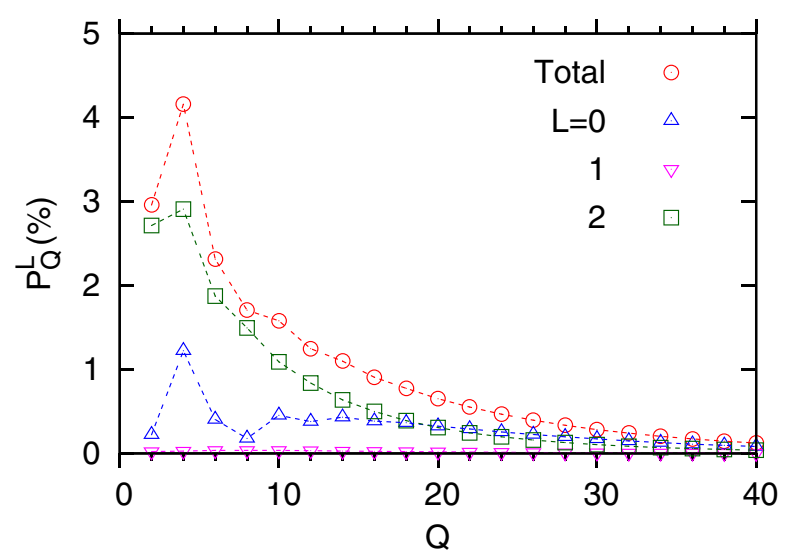

FIG. 3. (Color online) Angular-momentum decomposition of the occupation probability of the total $\mathrm{HO}$ quanta for the ground state of ${ }^{4}$ He. $P_{0}^{L}$ values are $79.1 \%, 0 \%, 0 \%$ for $L=0,1,2$, respectively. The $\mathrm{AV}^{\prime}+3 \mathrm{NF}$ potential is used.

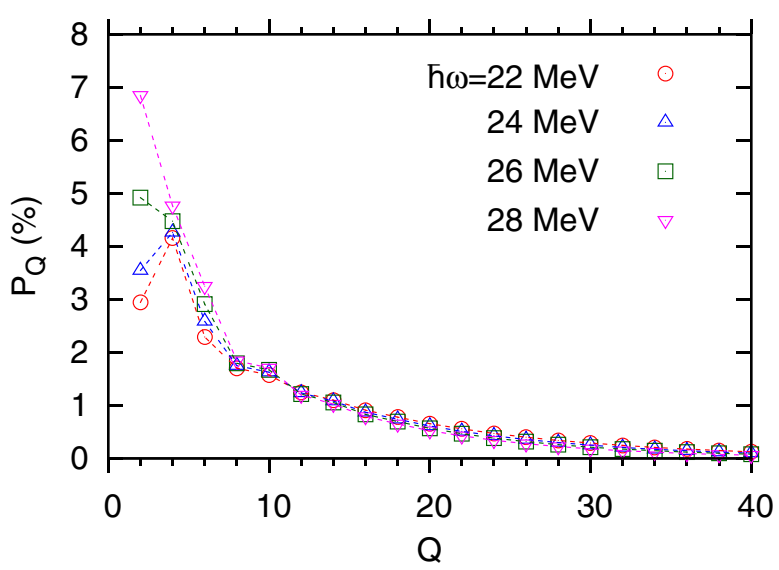

FIG. 4. (Color online) $\hbar \omega$ dependence of the occupation probability of the total $\mathrm{HO}$ quanta with $Q>0$ for the ground state of ${ }^{4} \mathrm{He}$. The AV8' +3 NF potential is used.

tail behavior in the $L=0$ component is due to the short-range repulsion. Both the tensor and short-range characters of the $N N$ potential make the convergence of conventional shellmodel calculations very slow.

Figure 4 presents how the probability distribution changes with different $\hbar \omega$ values. Although $P_{2}$ depends on $\hbar \omega$, the dependence of the sum of $P_{0}$ and $P_{2}$ is much weaker. This is understood as follows: Since the main role of the configurations with $Q=0$ and 2 is considered to describe the mean-field correlation of the system, each $P_{Q}$ value may depend on a choice of $\hbar \omega$ but the sum of them may not so much. A weaker dependence of $P_{Q}$ at $Q=4$ and 6 reflects the dominance of the tensor correlations. Finally, no $\hbar \omega$ dependence is found for $Q>6$. The higher- $Q$ components are always present and remain unchanged for different choices of $\hbar \omega$.

The mechanism responsible for enhancing the high- $Q$ components is different for the short-range repulsion and the tensor correlations of the realistic $N N$ interaction. The total number of $\mathrm{HO}$ quanta $Q$ is a sum of the $\mathrm{HO}$ quanta, $\sum_{i=1}^{N}\left(2 n_{i}+l_{i}\right)$, where $n_{i}$ and $l_{i}$ are respectively the principal and azimuthal quantum numbers of the $\mathrm{HO}$ wave function of the $i$ th nucleon. Since no spurious c.m. motion is included, the sum ranges over all the nucleons. As shown in Refs. [12-14], the short-range repulsion makes a strong depression at short distances in the pair correlation function. In the HO expansion, this depression of the pairwise relative wave functions at short distances is taken care of by superposing many HO wave functions that have larger $n_{i}$ with the same $l_{i}$, which obviously leads to the large- $Q$ components. On the other hand, the tensor correlations induce high- $Q$ components, because of the couplings between the $\mathrm{HO}$ wave functions with different $l_{i}$.

The $P_{Q}$ distribution actually reflects the momentum distribution. As we have already mentioned, the realistic interaction demands HO functions with large $Q$ in the coordinate space. Noting that the Fourier transform of the HO function in the coordinate space is again the $\mathrm{HO}$ function in the momentum space, the HO functions with large $Q$ certainly contain largemomentum components. References [10,14,30,31] showed that the momentum distribution has a long tail due to the tensor and short-range correlations. The HO functions with 
large $Q$ play a role in enhancing the high-momentum tail of the momentum distribution, whereas those with small $Q$ describe the mean-field structure below the Fermi momentum.

Since the inclusion of all the high- $Q$ components is not practical for heavier nuclei, an effective interaction starting from the realistic $N N$ interaction is usually employed to accelerate the convergence. Such effective interactions are derived in several approaches; for example, the Lee-Suzuki transformation [32], unitary correlation operator method (UCOM) [33,34], and similarity renormalization group [35]. A softened interaction always improves the energy convergence $[36,37]$ and succeeds to reproduce some low-lying spectra of light nuclei. See Ref. [1] for many such applications in the NCSM framework.

\section{B. First excited state of ${ }^{4} \mathrm{He}$ : cluster correlation}

The $P_{Q}$ distribution of the excited state of ${ }^{4} \mathrm{He}$ shows a pattern quite different from that of the ground state. Figure 5 plots $P_{Q}$ of the first-excited $J^{\pi} T=0^{+} 0$ state calculated with the four interaction models. The $P_{0}$ value almost vanishes, obviously because the state is orthogonal to the ground state whose major configuration is $(0 s)^{4}$. The distribution is less interaction-dependent at $Q<10$ compared to that of the ground state, which appears to be attributed to the weakly bound $3 N+N$ cluster structure of the first-excited state $[15,16]$. Assuming that the scattering length between $3 N$ and $N$ is much larger than its effective range, the system does not depend much on the detail of the interaction. This universal property is found in atomic systems and its similarity to the first-excited $0^{+} 0$ state is discussed in Ref. [38]. Beyond $Q=10, P_{Q}$ decreases monotonically and very slowly with increasing $Q$, and the values of $M_{Q}$ and $\sigma_{Q}$ in the case of the $\mathrm{AV}^{\prime}+3 \mathrm{NF}$ potential turn out to be 15.3 and 13.3, respectively. Appreciable probability still exists even at $Q=$ 30 , which is too large for standard shell-model calculations to incorporate [39]. From the angular-momentum decomposition of $P_{Q}$ we find out that the $L=0$ component, $P_{Q}^{0}$, dominates over the whole $Q$ region. This is also consistent with the fact that the $0_{2}^{+}$state has an $S$-wave $3 N+N$ cluster structure. If a state has a cluster structure, its $P_{Q}$ distribution spreads over

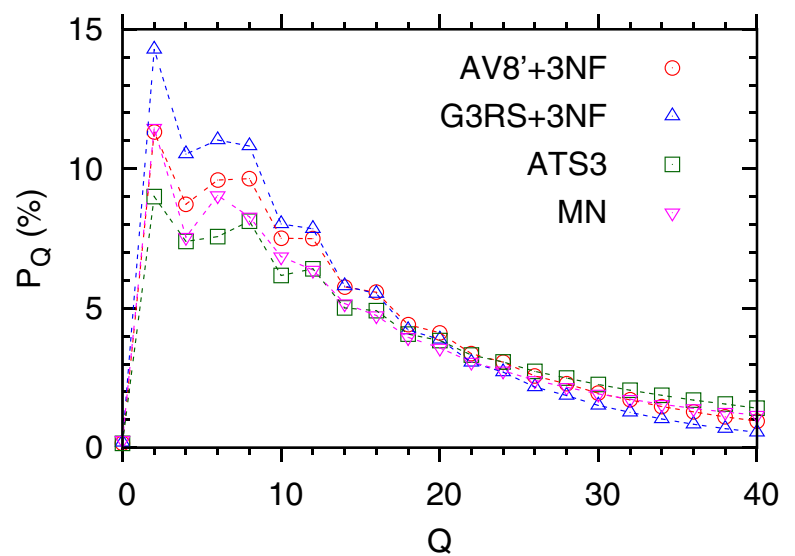

FIG. 5. (Color online) Occupation probability distributions of the total $\mathrm{HO}$ quanta for the first-excited state of ${ }^{4} \mathrm{He}$.

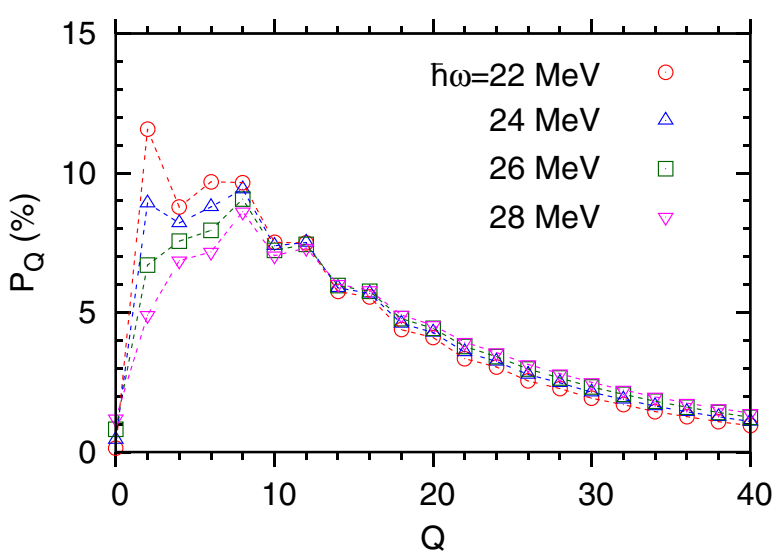

FIG. 6. (Color online) $\hbar \omega$ dependence of the occupation probability of the total $\mathrm{HO}$ quanta for the first-excited state of ${ }^{4} \mathrm{He}$. The $\mathrm{AV}^{\prime}+3 \mathrm{NF}$ potential is used.

large $Q$ because describing the relative motion between the clusters up to the asymptotic region requires configurations with large $Q$, even though the intrinsic wave functions of the clusters do not contain high HO excitations [5]. Other well-known examples, which support this fact, include the Hoyle state of ${ }^{12} \mathrm{C}[5,6]$ and the first-excited state of ${ }^{16} \mathrm{O}[4,7]$.

One may think that the first-excited state of ${ }^{4} \mathrm{He}$ can be described well in a shell model by choosing $\hbar \omega$ appropriately. To examine this question more closely, we exhibit the $\hbar \omega$ dependence of $P_{Q}$ in Fig. 6. The probability for $Q<10$ depends on $\hbar \omega$, but no practical dependence is found beyond this $Q$ value. Since the occupation probability is still significant for $Q>8$, we conclude that no appropriate choice for $\hbar \omega$ exists to describe the cluster state in the conventional shell-model truncation. Since the maximum major shell in shell-model calculations cannot be taken sufficiently large at present, it is reasonable to improve the wrong asymptotic behavior of the $\mathrm{HO}$ basis by combining with some other methods such as the resonating-group method [40,41].

\section{Inversion doublets in ${ }^{4} \mathrm{He}$}

As shown in Ref. [16], the first-excited state of ${ }^{4} \mathrm{He}$ has those negative-parity partners that have basically the same intrinsic structure. If a system has a two-cluster structure consisting of asymmetric subsystems, both positive- and negative-parity states may be found around the relevant threshold energy. A well-known example is ${ }^{16} \mathrm{O}$ with a ${ }^{12} \mathrm{C}+\alpha$ structure [4,7]. As a "mini" version of ${ }^{16} \mathrm{O}$, the spectrum of ${ }^{4} \mathrm{He}$ has some similarity to that of ${ }^{16} \mathrm{O}$. Because of the spin-isospin coupling of $3 N+N$ clusters, seven negative-parity states appear in ${ }^{4} \mathrm{He}$ above the first-excited $0^{+} 0$ state, as shown in calculations with the AV8' $+3 \mathrm{NF}$ potential [28].

Figure 7 plots the $P_{Q}$ values of the $0^{-} 0(21.01 \mathrm{MeV})$ and $2^{-} 0(21.84 \mathrm{MeV})$ states that are identified as the parityinverted partners [16]. For the sake of comparison, the $P_{Q}$ of the positive-parity partner, the $0_{2}^{+} 0$ state, is also drawn. Although the HO occupation probability is widely distributed to high $Q$ values, the $P_{1}$ value of the $0^{-}$state is $37.9 \%$, and the $M_{Q}$ and $\sigma_{Q}$ values are 5.42 and 6.36, respectively. These values are not as large as the corresponding values 


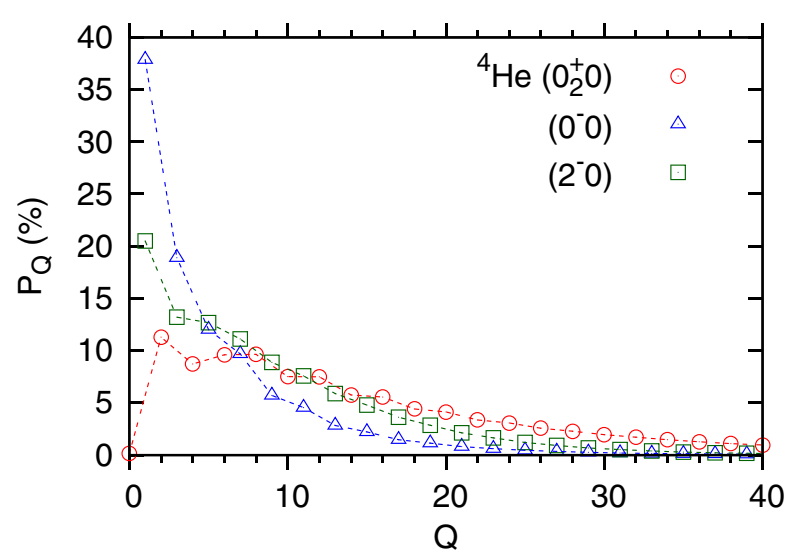

FIG. 7. (Color online) Occupation probability distributions of the HO quanta for the excited states of ${ }^{4} \mathrm{He}$. The AV8 ${ }^{\prime}+3 \mathrm{NF}$ potential is used.

for the $0_{2}^{+} 0$ state. Since it has a significant overlap with the $1 \mathrm{p}-1 \mathrm{~h}$ configurations, the $0^{-} 0$ state is expected to be described fairly well in large-scale shell-model calculations. Compared to the $0^{-} 0$ state, the $P_{Q}$ distribution of the $2^{-} 0$ state is closer to that of the $0^{+} 0$ state: the $P_{1}$ value is $20.5 \%$, while the $M_{Q}$ and $\sigma_{Q}$ values grow to 8.72 and 8.00 , respectively. Because of the $P$-wave centrifugal barrier between $3 N$ and $N$ clusters, the $2^{-} 0$ state shrinks compared with the $0_{2}^{+} 0$ state, and consequently the distribution of $P_{Q}$ is shifted to lower $Q$ values than that of the $0_{2}^{+} 0$ state.

\section{SUMMARY}

We have formulated a method for calculating the occupation probability of the number of total harmonic-oscillator (HO) quanta $Q$ to shed light on various types of nuclear correlations. We have analyzed the occupation probability distributions of the precise wave functions of $s$-shell nuclei that are obtained in the correlated Gaussian basis employing four kinds of interactions.

The HO probability distributions show quite different behavior, reflecting the characteristics of the interaction employed. In the case of the ground state of ${ }^{4} \mathrm{He}$, the tensor force significantly enhances the probability below $Q=20$. The short-range repulsion also plays an important role in mixing configurations with more than $Q=10$ excitations. For the excited states of ${ }^{4} \mathrm{He}$, the occupation probability is widely distributed to large $Q$ values and does not depend so much on the detail of the interaction. Configurations with a higher number of $\mathrm{HO}$ quanta are needed to describe the tail of the relative motion between the $3 N$ and $N$ clusters. In conformity with the parity-inverted doublet structure, the similarity of the $\mathrm{HO}$ distribution of the first-excited $J^{\pi} T=0^{+} 0$ state to that of the negative-parity excited states with $0^{-} 0$ and $2^{-} 0$ is discussed.

We find that all the probability distributions beyond $Q=10$ are insensitive to the choice for the $\mathrm{HO}$ oscillator frequency $\hbar \omega$. These high- $Q$ components in the wave function always exist irrespective of whether the interaction is effective or realistic and thereby lead to the difficulty in convergence or the extremely slow convergence in describing the cluster structure in the HO basis. The analysis presented here is useful for confirming that the occupation probability distribution in fact reflects important correlations and various kinds of structure of the nuclear wave functions. This analysis will be useful for providing a hint on how to develop an improved truncation scheme for huge shell-model spaces.

\section{ACKNOWLEDGMENTS}

The authors are greatly indebted to K. D. Launey for her careful reading of the manuscript. This work was supported in part by JSPS KAKENHI Grants No. 24540261 and No. 25800121 .

\section{APPENDIX: MATRIX ELEMENT FOR PROJECTION OPERATOR OF NUMBER OF HO QUANTA}

We define the Jacobi coordinate and the corresponding reduced mass as

$$
\boldsymbol{x}_{i}=\frac{1}{i+1} \sum_{j=1}^{i} \boldsymbol{r}_{j}-\boldsymbol{r}_{i+1}, \quad \mu_{i}=\frac{i}{i+1} m,
$$

with $i=1, \ldots, N-1$, where $\boldsymbol{r}_{j}$ is the $j$ th nucleon coordinate. Letting $\boldsymbol{\pi}_{i}$ denote the momentum conjugate to $\boldsymbol{x}_{i}$, the HO Hamiltonian $H_{\mathrm{HO}}(i)$ in Eq. (2) reads

$$
H_{\mathrm{HO}}(i)=\frac{\pi_{i}^{2}}{2 \mu_{i}}+\frac{1}{2} \mu_{i} \omega^{2} \boldsymbol{x}_{i}^{2} .
$$

We evaluate the matrix element of $e^{i \theta \mathcal{O}}$ between the generating functions of the CG (3) in three steps. First, we rewrite the generating function in a multiple-integral form of a product of Gaussian wave packets. Next, we act with $e^{i \theta \mathcal{O}}$ on the Gaussian wave packets. Finally, the multiple integral is performed analytically, which leads to the required matrix element.

Let $\psi_{\boldsymbol{R}_{i}}^{\gamma_{i}}\left(\boldsymbol{x}_{i}\right)$ denote a Gaussian wave packet centered at $\boldsymbol{R}_{i}$ with a width parameter $\gamma_{i}$ :

$$
\psi_{\boldsymbol{R}_{i}}^{\gamma_{i}}\left(\boldsymbol{x}_{i}\right)=\left(\frac{\gamma_{i}}{\pi}\right)^{3 / 4} \exp \left[-\frac{\gamma_{i}}{2}\left(\boldsymbol{x}_{i}-\boldsymbol{R}_{i}\right)^{2}\right] .
$$

The first step is to use the identity [8]

$$
\begin{aligned}
g(\boldsymbol{s} ; A, \boldsymbol{x})= & {\left[\frac{(\operatorname{det} \Gamma)^{3}}{(4 \pi)^{N-1}[\operatorname{det}(\Gamma-A)]^{2}}\right]^{\frac{3}{4}} } \\
& \times \exp \left[-\frac{1}{2} \tilde{\boldsymbol{s}}(\Gamma-A)^{-1} \boldsymbol{s}\right] \\
& \times \int d \boldsymbol{R} g\left(\Gamma(\Gamma-A)^{-1} \boldsymbol{s} ; A(\Gamma-A)^{-1} \Gamma, \boldsymbol{R}\right) \\
& \times \prod_{i=1}^{N-1} \psi_{\boldsymbol{R}_{i}}^{\gamma_{i}}\left(\boldsymbol{x}_{i}\right),
\end{aligned}
$$

where $\boldsymbol{R}$ stands for an $(N-1)$-dimensional column vector whose $i$ th element is $\boldsymbol{R}_{i}$ and $d \boldsymbol{R}=d \boldsymbol{R}_{1} d \boldsymbol{R}_{2} \cdots d \boldsymbol{R}_{N-1}$. $\Gamma$ is an $(N-1) \times(N-1)$ diagonal matrix whose element is chosen to be

$$
\Gamma_{i j}=\gamma_{i} \delta_{i, j}=\frac{\mu_{i} \omega}{\hbar} \delta_{i, j}
$$


The second step is to use the identity [see Eq. (5) of Ref. [5]], which makes it possible to obtain

$$
\begin{aligned}
& \exp \left\{i \theta\left[\frac{1}{\hbar \omega} H_{\mathrm{HO}}(j)-\frac{3}{2}\right]\right\} \psi_{\boldsymbol{R}_{j}}^{\gamma_{j}}\left(\boldsymbol{x}_{j}\right) \\
& \quad=\exp \left[-\frac{\gamma_{j}}{4}\left(1-z^{2}\right) \boldsymbol{R}_{j}^{2}\right] \psi_{z \boldsymbol{R}_{j}}^{\gamma_{j}}\left(\boldsymbol{x}_{j}\right)
\end{aligned}
$$

where $z=e^{i \theta}$. The operation of $e^{i \theta \mathcal{O}}$ on the product of the Gaussian wave packets is then given in a simple form:

$$
\begin{aligned}
e^{i \theta \mathcal{O}} \prod_{i=1}^{N-1} \psi_{\boldsymbol{R}_{i}}^{\gamma_{i}}\left(\boldsymbol{x}_{i}\right)= & \exp \left[-\frac{1}{4}\left(1-z^{2}\right) \tilde{\boldsymbol{R}} \Gamma \boldsymbol{R}\right]\left(\frac{\operatorname{det} \Gamma}{\pi^{N-1}}\right)^{3 / 4} \\
& \times \exp \left(-\frac{1}{2} \tilde{\boldsymbol{x}} \Gamma \boldsymbol{x}+z \tilde{\boldsymbol{R}} \Gamma \boldsymbol{x}-\frac{1}{2} z^{2} \tilde{\boldsymbol{R}} \Gamma \boldsymbol{R}\right) .
\end{aligned}
$$

The third step for obtaining $e^{i \theta \mathcal{O}} g(\boldsymbol{s} ; A, \boldsymbol{x})$ is to substitute the above result into Eq. (A4) and integrate over $\boldsymbol{R}$, which leads to the following compact result expressed again in terms of the generating function (3) of CG:

$$
\begin{aligned}
e^{i \theta \mathcal{O}} g(\boldsymbol{s} ; A, \boldsymbol{x})= & \left(\frac{\operatorname{det} \Gamma}{\operatorname{det} C}\right)^{3 / 2} \exp \left(-\frac{1}{2} \tilde{\boldsymbol{s}} G \boldsymbol{s}\right) \\
& \times g\left(z \Gamma C^{-1} \boldsymbol{s} ; D, \boldsymbol{x}\right),
\end{aligned}
$$

where the matrices $C, D$, and $G$ are given by

$$
\begin{aligned}
& C=A+\frac{1+z^{2}}{2}(\Gamma-A), \\
& D=\left[A+\frac{1-z^{2}}{2}(\Gamma-A)\right] C^{-1} \Gamma, \quad G=-\frac{1-z^{2}}{2} C^{-1} .
\end{aligned}
$$

It is easy to derive Eq. (4) using the result above.

A calculation of the matrix element of $e^{i \theta \mathcal{O}}$ between the CG (8) is therefore reduced to that of the overlap matrix element of the CG. See Refs. [9-11] that detail this process. An explicit form for the matrix element reads

$$
\begin{aligned}
& \left\langle F_{\left(L_{3} L_{4}\right) L M}\left(u_{3}, u_{4}, A^{\prime}, \boldsymbol{x}\right)\left|e^{i \theta \mathcal{O}}\right| F_{\left(L_{1} L_{2}\right) L M}\left(u_{1}, u_{2}, A, \boldsymbol{x}\right)\right\rangle \\
& \quad=\left(\frac{\operatorname{det} \Gamma \operatorname{det}\left(A+A^{\prime}\right)}{\operatorname{det} B \operatorname{det} C}\right)^{3 / 2}\left\langle F_{\left(L_{3} L_{4}\right) L M}\left(u_{3}, u_{4}, A^{\prime}, \boldsymbol{x}\right)\left|F_{\left(L_{1} L_{2}\right) L M}\left(u_{1}, u_{2}, A, \boldsymbol{x}\right)\right|_{\rho_{i j} \rightarrow X_{i j}},\right.
\end{aligned}
$$

where $\left\langle F_{\left(L_{3} L_{4}\right) L M}\left(u_{3}, u_{4}, A^{\prime}, \boldsymbol{x}\right) \mid F_{\left(L_{1} L_{2}\right) L M}\left(u_{1}, u_{2}, A, \boldsymbol{x}\right)\right\rangle$ is the overlap matrix element [see Eq. (B.10) of Ref. [10]] and the $\rho_{i j}=\tilde{u}_{i}\left(A+A^{\prime}\right)^{-1} u_{j}$, which appears in Ref. [10], should be replaced by $X_{i j}$ which are defined as follows:

$$
X_{12}=\tilde{u}_{1}\left(\frac{1-z^{2}}{2} C^{-1}+z^{2} C^{-1} \Gamma G^{-1} \Gamma C^{-1}\right) u_{2},
$$

$$
\begin{aligned}
& X_{13}=z \tilde{u}_{1} C^{-1} \Gamma G^{-1} u_{3}, \quad X_{14}=z \tilde{u}_{1} C^{-1} \Gamma G^{-1} u_{4}, \\
& X_{23}=z \tilde{u}_{2} C^{-1} \Gamma G^{-1} u_{3}, \quad X_{24}=z \tilde{u}_{2} C^{-1} \Gamma G^{-1} u_{4}, \\
& X_{34}=\tilde{u}_{3} G^{-1} u_{4} .
\end{aligned}
$$

[1] B. R. Barrett, P. Navrátil, and J. P. Vary, Prog. Part. Nucl. Phys. 69, 131 (2013), and references therein.

[2] M. Włoch, D. J. Dean, J. R. Gour, M. Hjorth-Jensen, K. Kowalski, T. Papenbrock, and P. Piecuch, Phys. Rev. Lett. 94, 212501 (2005).

[3] P. Maris, J. P. Vary, and A. M. Shirokov, Phys. Rev. C 79, 014308 (2009).

[4] Y. Suzuki, Prog. Theor. Phys. 55, 1751 (1976); 56, 111 (1976).

[5] Y. Suzuki, K. Arai, Y. Ogawa, and K. Varga, Phys. Rev. C 54, 2073 (1996).

[6] T. Neff, J. Phys.: Conf. Ser. 403, 012028 (2012).

[7] W. Horiuchi and Y. Suzuki, Phys. Rev. C 89, 011304(R) (2014).

[8] K. Varga and Y. Suzuki, Phys. Rev. C 52, 2885 (1995).

[9] Y. Suzuki and K. Varga, Stochastic Variational Approach to Quantum-Mechanical Few-Body Problems, Lecture Notes in Physics (Springer, Berlin, 1998), Vol. m54.

[10] Y. Suzuki, W. Horiuchi, M. Orabi, and K. Arai, Few-Body Syst. 42, 33 (2008).

[11] S. Aoyama, K. Arai, Y. Suzuki, P. Descouvemont, and D. Baye, Few-Body Syst. 52, 97 (2012).
[12] H. Kamada et al., Phys. Rev. C 64, 044001 (2001).

[13] J. L. Forest, V. R. Pandharipande, S. C. Pieper, R. B. Wiringa, R. Schiavilla, and A. Arriaga, Phys. Rev. C 54, 646 (1996).

[14] H. Feldmeier, W. Horiuchi, T. Neff, and Y. Suzuki, Phys. Rev. C 84, 054003 (2011).

[15] E. Hiyama, B. F. Gibson, and M. Kamimura, Phys. Rev. C 70, 031001(R) (2004).

[16] W. Horiuchi and Y. Suzuki, Phys. Rev. C 78, 034305 (2008).

[17] R. Roth and P. Navrátil, Phys. Rev. Lett. 99, 092501 (2007).

[18] C. Forssén, R. Roth, and P. Navrátil, J. Phys. G: Nucl. Part. Phys. 40, 055105 (2013).

[19] T. Dytrych, K. D. Sviratcheva, C. Bahri, J. P. Draayer, and J. P. Vary, Phys. Rev. Lett. 98, 162503 (2007).

[20] N. Shimizu, T. Abe, Y. Tsunoda, Y. Utsuno, T. Yoshida, T. Mizusaki, M. Honma, and T. Otsuka, Prog. Theor. Exp. Phys. 2012, 01A205 (2012).

[21] T. Myo, S. Sugimoto, K. Kato, H. Toki, and K. Ikeda, Prog. Theor. Phys. 117, 257 (2007).

[22] D. R. Thompson, M. LeMere, and Y. C. Tang, Nucl. Phys. A 286, 53 (1977). 
[23] I. R. Afnan and Y. C. Tang, Phys. Rev. 175, 1337 (1968).

[24] R. Tamagaki, Prog. Theor. Phys. 39, 91 (1968).

[25] B. S. Pudliner, V. R. Pandharipande, J. Carlson, S. C. Pieper, and R. B. Wiringa, Phys. Rev. C 56, 1720 (1997).

[26] J. Mitroy, S. Bubin, W. Horiuchi, Y. Suzuki, L. Adamowicz, W. Cencek, K. Szalewicz, J. Komasa, D. Blume, and K. Varga, Rev. Mod. Phys. 85, 693 (2013).

[27] D. R. Tilley, H. R. Weller, and G. M. Hale, Nucl. Phys. A 541, 1 (1992).

[28] W. Horiuchi and Y. Suzuki, Phys. Rev. C 87, 034001 (2013).

[29] W. Horiuchi and Y. Suzuki, Few-Body Syst. 54, 2407 (2013).

[30] R. Schiavilla, R. B. Wiringa, S. C. Pieper, and J. Carlson, Phys. Rev. Lett. 98, 132501 (2007).

[31] W. Horiuchi and Y. Suzuki, Phys. Rev. C 76, 024311 (2007).

[32] K. Suzuki and S. Y. Lee, Prog. Theor. Phys. 64, 2091 (1980).
[33] H. Feldmeier, T. Neff, R. Roth, and J. Schnack, Nucl. Phys. A 632, 61 (1998).

[34] T. Neff and H. Feldmeier, Nucl. Phys. A 713, 311 (2003).

[35] S. K. Bogner, R. J. Furnstahl, and R. J. Perry, Phys. Rev. C 75, 061001 (2007).

[36] R. Roth, T. Neff, and H. Feldmeier, Prog. Part. Nucl. Phys. 65, 50 (2010).

[37] S. K. Bogner, R. J. Furnstahl, and A. Schwenk, Prog. Part. Nucl. Phys. 65, 94 (2010).

[38] E. Hiyama and M. Kamimura, Phys. Rev. A 85, 062505 (2012).

[39] E. D. Jurgenson, P. Navrátil, and R. J. Furnstahl, Phys. Rev. C 83, 034301 (2011).

[40] S. Baroni, P. Navrátil, and S. Quaglioni, Phys. Rev. Lett. 110, 022505 (2013); Phys. Rev. C 87, 034326 (2013).

[41] S. Quaglioni, C. Romero-Redondo, and P. Navrátil, Phys. Rev. C 88, 034320 (2013). 